PROCEEDINGS OF THE

AMERICAN MATHEMATICAL SOCIETY

Volume 134, Number 7 , Pages 1875-1880

S 0002-9939(05)08348-6

Article electronically published on December 19, 2005

\title{
ON HOMEOMORPHISMS AND QUASI-ISOMETRIES OF THE REAL LINE
}

\author{
PARAMESWARAN SANKARAN \\ (Communicated by Alexander N. Dranishnikov)
}

\begin{abstract}
We show that the group of piecewise-linear homeomorphisms of $\mathbb{R}$ having bounded slopes surjects onto the group $Q I(\mathbb{R})$ of all quasi-isometries of $\mathbb{R}$. We prove that the following groups can be imbedded in $Q I(\mathbb{R})$ : the group of compactly supported piecewise-linear homeomorphisms of $\mathbb{R}$, the Richard Thompson group $F$, and the free group of continuous rank.
\end{abstract}

\section{INTRODUCTION}

We begin by recalling the notion of quasi-isometry. Let $f: X \longrightarrow X^{\prime}$ be a map (which is not assumed to be continuous) between metric spaces. We say that $f$ is a $C$-quasi-isometric embedding if there exists a $C>1$ such that

$$
C^{-1} d(x, y)-C \leq d^{\prime}(f(x), f(y)) \leq C d(x, y)+C
$$

for all $x, y \in X$. Here $d, d^{\prime}$ denote the metrics on $X, X^{\prime}$, respectively. If, further, every $x^{\prime} \in X^{\prime}$ is within distance $C$ from the image of $f$, we say that $f$ is a $C$-quasi isometry. If $f$ is a quasi-isometry (for some $C$ ), then there exists a quasi-isometry $f^{\prime}: X^{\prime} \longrightarrow X$ (for a possibly different constant $C^{\prime}$ ) such that $f^{\prime} \circ f$ (resp. $f \circ f^{\prime}$ ) is quasi-isometry equivalent to the identity map of $X$ (resp. $X^{\prime}$ ). (Two maps $f, g: X \longrightarrow X$ are said to be quasi-isometrically equivalent if there exists a constant $M$ such that $d(f(x), g(x)) \leq M$ for all $x \in X$.) Let $[f]$ denote the equivalence class of a quasi-isometry $f: X \longrightarrow X$. The set $Q I(X)$ of all equivalence classes of quasiisometries of $X$ is a group under composition: $[f] \cdot[g]=[f \circ g]$ for $[f],[g] \in Q I(X)$. If $X^{\prime}$ is quasi-isometry equivalent to $X$, then $Q I\left(X^{\prime}\right)$ is isomorphic to $Q I(X)$. We refer the reader to [1] for basic facts concerning quasi-isometry. For example $t \mapsto[t]$ is a quasi-isometry from $\mathbb{R}$ to $\mathbb{Z}$.

Let $f: \mathbb{R} \longrightarrow \mathbb{R}$ be any homeomorphism of $\mathbb{R}$. Denote by $B(f)$ the set of break points of $f$, i.e., points where $f$ fails to have derivative and by $\Lambda(f)$ the set of slopes of $f$, i.e., $\Lambda(f)=\left\{f^{\prime}(t) \mid t \in \mathbb{R} \backslash B(f)\right\}$. Note that $B(f) \subset \mathbb{R}$ is discrete if $f$ is piecewise differentiable.

Definition 1.1. We say that a subset $\Lambda$ of $\mathbb{R}^{*}$, the set of non-zero real numbers, is bounded if there exists an $M>1$ such that $M^{-1}<|\lambda|<M$ for all $\lambda \in \Lambda$. We say that a homeomorphism $f$ of $\mathbb{R}$ which is piecewise differentiable has bounded slopes if $\Lambda(f)$ is bounded.

Received by the editors October 4, 2004 and, in revised form, February 8, 2005.

2000 Mathematics Subject Classification. Primary 20F65, 20F28; Secondary 20F67.

Key words and phrases. PL-homeomorphisms, quasi-isometry, Thompson's group, free groups.

(C)2005 American Mathematical Society Reverts to public domain 28 years from publication 
We denote by $P L_{\delta}(\mathbb{R})$ the set of all those piecewise-linear homeomorphisms $f$ of $\mathbb{R}$ such that $\Lambda(f)$ is bounded. It is clear that $P L_{\delta}(\mathbb{R})$ is a subgroup of the group $P L(\mathbb{R})$ of all piecewise-linear homeomorphisms of $\mathbb{R}$.

It is easy to see that each $f \in P L_{\delta}(\mathbb{R})$ is a quasi-isometry. (See Lemma 2.1 below.) One has a natural homomorphism $\varphi: P L_{\delta}(\mathbb{R}) \longrightarrow Q I(\mathbb{R})$, where $\varphi(f)=[f]$ for all $f \in P L_{\delta}(\mathbb{R})$.

Theorem 1.2. The natural homomorphism $\varphi: P L_{\delta}(\mathbb{R}) \longrightarrow Q I(\mathbb{R})$, defined as $f \mapsto$ $[f]$, is surjective.

If $f: \mathbb{R} \longrightarrow \mathbb{R}$ is a homeomorphism, recall that $\operatorname{Supp}(f)$, the support of $f$, is the closure of the set $\{x \in \mathbb{R} \mid f(x) \neq x\}$ of all points moved by $f$. Denote by $P L_{\kappa}(\mathbb{R})$ the group of all piecewise-linear homeomorphisms of $\mathbb{R}$ which have compact support. It is obvious that $P L_{\kappa}(\mathbb{R}) \subset \operatorname{ker}(\varphi)$.

Let $\Gamma$ be a group of homeomorphisms of $\mathbb{S}^{1}$. Any $f \in \Gamma$ can be lifted to obtain a homeomorphism $\widetilde{f}$ of $\mathbb{R}$ over the covering projection $p: \mathbb{R} \longrightarrow \mathbb{S}^{1}, t \mapsto \exp (2 \pi \sqrt{-1} t)$. The set $\widetilde{\Gamma}$ of all homeomorphisms of $\mathbb{R}$ which are lifts of elements of $\Gamma$ is a subgroup of the group Homeo $(\mathbb{R})$ of all homeomorphisms of $\mathbb{R}$. Indeed $\widetilde{\Gamma}$ is a central extension of $\Gamma$ by the infinite cyclic group generated by translation by $1: x \mapsto x+1$. Denote by $\operatorname{Diff}\left(\mathbb{S}^{1}\right)$ the group of all $C^{\infty}$ diffeomorphisms of the circle. When $\Gamma$ is one of the groups $P L\left(\mathbb{S}^{1}\right)$, $\operatorname{Diff}\left(\mathbb{S}^{1}\right)$, any element of $\widetilde{f} \in \widetilde{\Gamma}$ has bounded slope and is quasi-isometrically equivalent to the identity map of $\mathbb{R}($ since $\widetilde{f}(x+n)=\widetilde{f}(x)+n$ for $n \in \mathbb{Z})$.

Recall that Richard Thompson discovered the group

$$
F=\left\langle x_{0}, x_{1}, \cdots \mid x_{i} x_{j} x_{i}^{-1}=x_{j+1}, i<j\right\rangle
$$

and used it in some constructions in logic related to word problems. The group $F$ is finitely presentable with two generators $x_{0}, x_{1}$ and two relations. This group and a closely related larger group $G$ have since then appeared in several contexts including homotopy theory [6, homological group theory [3], Teichmüller theory [9], etc. The group $F$ is isomorphic to the subgroup of piecewise-linear homeomorphisms of $\mathbb{R}$ which are the identity outside the unit interval $I$ such that $B(f)$ is contained in dyadic rationals and $\Lambda(f)$ is contained in the subgroup of $\mathbb{R}^{*}$ generated by 2 . Although $F$ satisfies no (non-trivial) group law, it contains no non-abelian free group. The group $G$ is the group of piecewise-linear homeomorphisms $f$ of the circle $\mathbb{S}^{1}=I /\{0,1\}$ with $B(\widetilde{f})$ contained in dyadic rationals and $\Lambda(\widetilde{f})$ contained in the multiplicative subgroup of $\mathbb{R}^{*}$ generated by 2 for some lift $\tilde{f}$ of $f$. It is the first known example of a finitely presented infinite simple group. We recommend the beautiful survey article 4 for further information about Richard Thompson's groups.

We shall prove the following theorem:

Theorem 1.3. The following groups can be imbedded in $Q I(\mathbb{R})$ :

(i) the groups $\widetilde{\operatorname{Diff}}\left(\mathbb{S}^{1}\right)$ and $\widetilde{P L}\left(\mathbb{S}^{1}\right)$,

(ii) the group $P L_{\kappa}(\mathbb{R})$,

(iii) the Thompson's group $F$, and

(iv) the free group of rank $c$, the continuum.

Our proofs are completely elementary. We explain the main idea of the proof of Theorem 1.3. Take for example the group $P L_{\kappa}(\mathbb{R})$. The first step is to realise 
this as a subgroup $\Gamma_{1}$ of $P L_{\kappa}(\mathbb{R})$ having support in $(0,1)$. This is easily achieved by imbedding $\mathbb{R}$ in the interval $(0,1)$. The group $\Gamma_{1}$ can be thought of as a group of piecewise-linear homeomorphisms of the circle. Lifting this back to $\mathbb{R}$ via the covering projection, we now obtain a group $\widetilde{\Gamma}_{1}$ which no longer has compact support. However each element of this group is quasi-isometric to $i d$. So we conjugate this group by a piecewise-linear homeomorphism whose slope grows exponentially. The result is that the features of each element of $\widetilde{\Gamma}_{1}$ get magnified, resulting in a quasiisometry not representing 1 . The same trick works for $\widetilde{\operatorname{Diff}}\left(\mathbb{S}^{1}\right)$ as well. Parts (iii) and (iv) follow from known embeddings of the relevant groups.

\section{Proof of Theorem 1.2}

We first establish the following basic observation.

Lemma 2.1. Let $f$ be a piecewise differentiable homeomorphism of $\mathbb{R}$ with $\Lambda(f) \subset$ $\mathbb{R}^{*}$ bounded. Then $f$ is a quasi-isometry.

Proof. Replacing $f$ by $-f$ if necessary, one may assume without loss of generality that $f$ is monotone increasing.

Suppose that $\Lambda(f) \subset(1 / M, M)$. If $f$ is differentiable everywhere, then it is an $M$-quasi-isometry.

Suppose that $B(f) \neq \emptyset$. Let $a \in \mathbb{R}$. For any $b>a$, let $a_{1}<\cdots<a_{k}$ be the points of $(a, b)$ where $f$ is non-differentiable. Then, applying the mean value theorem, $f(b)-f(a)=\sum_{0 \leq i \leq k}\left(f\left(a_{i+1}\right)-f\left(a_{i}\right)\right)=\sum_{0 \leq i \leq k} f^{\prime}\left(c_{i}\right)\left(a_{i+1}-a_{i}\right)$ for some $c_{i} \in\left(a_{i}, a_{i+1}\right)$. Since $\Lambda(f) \subset(1 / M, M)$, it follows that $M^{-1}(b-a)<f(b)-f(a)<$ $M(b-a)$. Since $a, b \in \mathbb{R}$ are arbitrary, we conclude that $f$ is a quasi-isometry.

One has a well-defined map $\varphi: P L_{\delta}(\mathbb{R}) \longrightarrow Q I(\mathbb{R})$ which is a homomorphism. We now prove that $\varphi$ is surjective.

Lemma 2.2. Let $f: \mathbb{R} \longrightarrow \mathbb{R}$ be a $C$-quasi-isometry that preserves the ends of $\mathbb{R}$. Let $x \in \mathbb{R}$. Then (i) there exists $y$ such that $y-x \leq 4 C^{2}$ is positive integer and $f(y)>f(x)$; (ii) there exists $v$ such that $x-v \leq 4 C^{2}$ is a positive integer and $f(x)>f(v)$.

Proof. If $f(x+1)>f(x)$, then $y=x+1$ meets our requirements.

Assume that $f(x)>f(y)$ for all $y$ such that $x+1 \leq y<4 C^{2}+x$. Let $z \geq x+2$ be the smallest real number such that $z-x$ is a positive integer and $f(z)>f(x) \geq$ $f(z-1)$. Such a $z$ exists since $f(t) \rightarrow+\infty$ as $t \rightarrow+\infty$. By our assumption $z-x \geq 4 C^{2}+1$. Set $u=z-1$. Then the inequality $(*)$ implies $f(u)<f(x)+$ $C-C^{-1}(u-x) \leq f(x)-3 C$ and $f(z)-f(u)<C(z-u)+C=2 C$. Hence $f(z)<f(u)+2 C<f(x)-C$, i.e., $f(z)-f(x)<-C$. This contradicts our hypothesis that $f(z)>f(x)$, completing the proof of part (i). The proof of part (ii) is similar.

Proof of Theorem 1.2. Since the subgroup $Q I^{+}(\mathbb{R}) \subset Q I(\mathbb{R})$ that preserves the ends $\{+\infty,-\infty\}$ of $\mathbb{R}$ is of index 2 and since $P L_{\delta}(\mathbb{R})$ contains elements which are orientation reversing, it suffices to show that $Q I^{+}(\mathbb{R})$ is contained in the image of $\varphi$, where $Q I^{+}(\mathbb{R}) \subset Q I(\mathbb{R})$ is the index 2 subgroup whose elements preserve the ends of $\mathbb{R}$.

Let $f: \mathbb{R} \longrightarrow \mathbb{R}$ be a $C$-quasi isometry, with $C>1$, which preserves the ends of $\mathbb{R}$. We assume, as we may, that $C$ is a positive integer. 
Set $x_{0}=0$. We define $x_{k} \in \mathbb{Z}$ for any integer $k$ as follows: Let $k \geq 1$. Having defined $x_{k-1}$ inductively, choose $x_{k}>x_{k-1}$ to be the smallest integer such that $f\left(x_{k}\right)>f\left(x_{k-1}\right)$. For any negative integer $k$, we define $x_{k}$ analogously (by downward induction) as the greatest integer such that $x_{k}<x_{k+1}$ and $f\left(x_{k}\right)<f\left(x_{k+1}\right)$.

Set $y_{k}:=x_{C^{3} k}$, and let $B:=\left\{y_{k} \mid k \in \mathbb{Z}\right\} \subset \mathbb{Z}$. By Lemma 2.2, we see that $B$ is a discrete subset of $\mathbb{R}$ which is $4 C^{5}$-dense in $\mathbb{R}$. Note that for any $k \in \mathbb{Z}$, $y_{k}-y_{k-1} \geq C^{3}$.

Since $f\left(y_{k}\right)>f\left(y_{k-1}\right)$ for all $k \in \mathbb{Z}$, there exists a unique piecewise-linear homeomorphism $g: \mathbb{R} \longrightarrow \mathbb{R}$ such that $g\left(y_{k}\right)=f\left(y_{k}\right)$ and is linear on the interval $\left[y_{k-1}, y_{k}\right]$ for every $k \in \mathbb{Z}$. We claim that $g$ has bounded slopes. Since $g$ is linear on each of the intervals $\left[y_{k-1}, y_{k}\right]$, we need only bound $\frac{g\left(y_{k}\right)-g\left(y_{k-1}\right)}{y_{k}-y_{k-1}}$. Indeed,

$$
\frac{g\left(y_{k}\right)-g\left(y_{k-1}\right)}{y_{k}-y_{k-1}}=\frac{f\left(y_{k}\right)-f\left(y_{k-1}\right)}{y_{k}-y_{k-1}}<C+\frac{C}{y_{k}-y_{k-1}} \leq C+C^{-2}
$$

as $y_{k}-y_{k-1} \geq C^{3}$. Similarly,

$$
\frac{g\left(y_{k}\right)-g\left(y_{k-1}\right)}{y_{k}-y_{k-1}}>C^{-1}-C^{-2} .
$$

It follows that $\Lambda(g) \subset\left[C^{-1}-C^{-2}, C+C^{-2}\right]$ and $g \in P L_{\delta}(\mathbb{R})$.

Since $f$ and $g$ agree on the quasi-dense set $B$, we see that $[f]=[g]$. This completes the proof.

Remark 2.3. (i) By setting $g\left(y_{k}\right)$ equal to a rational number sufficiently close to $f\left(y_{k}\right)$ in the above proof, we see that since $y_{k} \in \mathbb{Z}$, the element $g \in P L_{\delta}(\mathbb{R})$ has rational slopes. Consequently it follows that $\varphi$ restricted to the subgroup $P L_{\mathbb{Q}}^{\mathbb{Q}^{*}}(\mathbb{R})$ of $P L_{\delta}(\mathbb{R})$ consisting of those $g \in P L_{\delta}(\mathbb{R})$ having slopes in $\mathbb{Q}^{*}$ and $B(g)$ contained in $\mathbb{Q}$ is surjective.

(ii) The kernel of $\varphi$ contains the group of all piecewise-linear homeomorphisms which have slope 1 outside a compact interval. This latter group equals the derived group $P L F^{\prime}(\mathbb{R})$, where $P L F(\mathbb{R})$ denotes the subgroup of $P L_{\delta}(\mathbb{R})$ consisting of homeomorphisms $f$ for which $B(f)$ is finite. Also $P L_{\kappa}(\mathbb{R})=P L F^{\prime \prime}(\mathbb{R})$. See [2].

\section{Proof of Theorem 1.3}

Let $h_{1}: \mathbb{R} \longrightarrow(0,1)$ be the homeomorphism defined by $h_{1}(-x)=1-h_{1}(x)$ for every $x \in \mathbb{R}, h_{1}(n)=1-1 /(n+2)$ for each integer $n \geq 0$, and is linear on each interval $[n, n+1]$ for $n \in \mathbb{Z}$. If $f$ is any compactly supported (piecewiselinear) homeomorphism of $\mathbb{R}$, then $h_{1} \circ f \circ h_{1}^{-1}$ is a compactly supported (piecewiselinear) homeomorphism of $(0,1)$. Since $\mathbb{S}^{1}=I /\{0,1\}$, we also get an embedding $\bar{\eta}: P L_{\kappa}(\mathbb{R}) \longrightarrow P L\left(\mathbb{S}^{1}\right)$, where $\bar{\eta}(f)$ is defined to be the extension of $h_{1} \circ f \circ h_{1}^{-1}$ to $\mathbb{S}^{1}$. We define $\eta: P L_{\kappa}(\mathbb{R}) \longrightarrow P L_{\delta}(\mathbb{R})$ as the imbedding $f \mapsto \eta(f)$, where $\eta(f)(n)=n$ for $n \in \mathbb{Z}$ and $\eta(f)(x)=n+h_{1} f h_{1}^{-1}(x-n)$ for $n<x<n+1$.

Let $h_{0}: \mathbb{R} \longrightarrow \mathbb{R}$ be the piecewise-linear homeomorphism defined as follows: $h_{0}(-x)=-h_{0}(x) \forall x \in \mathbb{R}, h_{0}(x)=x$ for $0 \leq x \leq 1$ and maps the interval $[n, n+1]$ onto $\left[2^{n-1}, 2^{n}\right]$ linearly for each positive integer $n$.

Suppose $f: \mathbb{S}^{1} \longrightarrow \mathbb{S}^{1}$ is an orientation-preserving piecewise-linear homeomorphism or a diffeomorphism. Let $\tilde{f}: \mathbb{R} \longrightarrow \mathbb{R}$ be any lift of $f$ so that $p \circ \tilde{f}=f \circ p$, where $p: \mathbb{R} \longrightarrow \mathbb{S}^{1}$ is the covering projection $t \mapsto \exp (2 \pi \sqrt{-1} t)$. Then $[\widetilde{f}]=1$ in 
$Q I(\mathbb{R})$. (Indeed one has $\widetilde{f}(x+n)=n+\widetilde{f}(x)$ for all $x \in \mathbb{R}$ and $n \in \mathbb{Z}$ and so $|\widetilde{f}-i d| \leq|\widetilde{f}(0)|+1$.)

Let $\Gamma$ be one of the groups $P L\left(\mathbb{S}^{1}\right)$ or $\operatorname{Diff}\left(\mathbb{S}^{1}\right)$ and let $\widetilde{\Gamma}$ be the group of homeomorphisms of $\mathbb{R}$ which are lifts of elements of $\Gamma$ with respect to the covering projection $p$. For $\widetilde{f} \in \widetilde{\Gamma}$ set $f_{0}:=h_{0} \widetilde{f} h_{0}^{-1}$. Clearly, $\widetilde{f} \mapsto f_{0}$ is a monomorphism of groups $\widetilde{\Gamma} \longrightarrow \operatorname{Homeo}(\mathbb{R})$. We claim that for any $\widetilde{f} \in \widetilde{\Gamma}, f_{0}$ is a quasi-isometry. To see this, we assume without loss of generality that $\tilde{f}$ is orientation preserving. It is clear that $f_{0}$ is differentiable outside a discrete subset of $\mathbb{R}$. We claim that $f_{0}$ has bounded slopes. Since $f_{0}$ has continuous derivatives on each interval on which $f_{0}$ has derivatives, it suffices to show that the set $\left\{f_{0}^{\prime}(t)\right\}$ as $t$ varies in $\mathbb{R} \backslash B$ is bounded, where $B$ is any discrete set which contains $B\left(f_{0}\right)$. We set $B:=B\left(h_{0}\right) \cup h_{0} B(\widetilde{f}) \cup h_{0} \widetilde{f}^{-1} B\left(h_{0}\right)$.

Let $0<m<M$ be such that $m<\tilde{f}^{\prime}(x)<M$ for $x \in \mathbb{R}$. Let $t \in \mathbb{R} \backslash B$ and set $s=h_{0}^{-1}(t), u=\widetilde{f}(s)$ so that $h_{0}^{-1}, \widetilde{f}, h_{0}$ are differentiable at $t, s, u$, respectively. Consequently $f_{0}$ is differentiable at $t$.

Since $|u-s|=|\widetilde{f}(s)-s|<q$, where $q:=[|\widetilde{f}(0)|]+2$, we see that $2^{-q}<$ $h_{0}^{\prime}(u) / h_{0}^{\prime}(s)<2^{q}$. Using the chain rule, it follows that $f_{0}^{\prime}(t)=h_{0}^{\prime}(u) \widetilde{f}^{\prime}(s)\left(h_{0}^{-1}\right)^{\prime}(t)=$ $\tilde{f}^{\prime}(s) h_{0}^{\prime}(u) / h_{0}^{\prime}(s)$ lies in the interval $\left(2^{-q} m, 2^{q} M\right)$. It follows from Lemma 2.1 that $f_{0}$ is a quasi-isometry.

It is clear that the map $\psi: \widetilde{\Gamma} \longrightarrow Q I(\mathbb{R})$ defined as $\widetilde{f} \mapsto\left[f_{0}\right]$ is a homomorphism.

We are now ready to prove Theorem 1.3 .

Proof of Theorem 1.3. We use the above notations throughout the proof.

(i) We prove that $\psi: \widetilde{\Gamma} \longrightarrow Q I(\mathbb{R})$ is a monomorphism where $\Gamma=P L\left(\mathbb{S}^{1}\right)$ or $\operatorname{Diff}\left(\mathbb{S}^{1}\right)$. Suppose that $\widetilde{f} \in \widetilde{\Gamma}, \widetilde{f} \neq i d$. We shall show that that $\left|f_{0}-i d\right|$ is unbounded. Choose $x$ in the interval $[0,1)$ such that $\widetilde{f}(x) \neq x$. Set $k=[\widetilde{f}(x)]$ so that $\widetilde{f}(x)=k+y, 0 \leq y<1$. Replacing $\widetilde{f}$ by its inverse if necessary, we assume without loss of generality that $x<\widetilde{f}(x)$. This implies that $k \geq 0$ with equality only if $y>x$. For any positive integer $n$, we have $f_{0}\left(2^{n}+2^{n} x\right)=h_{0} \widetilde{f} h_{0}^{-1}\left(2^{n}+2^{n} x\right)=$ $h_{0} \widetilde{f}(n+1+x)=h_{0}(n+1+\widetilde{f}(x))=h_{0}(n+1+k+y)=2^{n+k}+2^{n+k} y$.

If $k=0$, then $y>x$ and so $f_{0}\left(2^{n}+2^{n} x\right)-\left(2^{n}+2^{n} x\right)=2^{n}(y-x)$. Thus $\left|f_{0}-i d\right|$ is unbounded.

If $k>0$, then $f_{0}\left(2^{n}+2^{n} x\right)-\left(2^{n}+2^{n} x\right)=2^{n+k}+2^{n+k} y-2^{n}-2^{n} x \geq 2^{n+1}-$ $2^{n}-2^{n} x=2^{n}(1-x)$. As $0 \leq x<1$, again it follows that $\left|f_{0}-i d\right|$ is unbounded.

(ii) As observed earlier, $\eta: P L_{\kappa}(\mathbb{R}) \longrightarrow P L_{\delta}(\mathbb{R})$ is a monomorphism. It is evident that the image of $\eta$ is contained in $\widetilde{P L}\left(\mathbb{S}^{1}\right)$. Since $\psi: \widetilde{P L}\left(\mathbb{S}^{1}\right) \longrightarrow Q I(\mathbb{R})$ is a monomorphism by (i), assertion (ii) follows.

(iii) Now statement (iii) follows from (ii) above and the fact that Thompson's group $F$ is isomorphic to the subgroup of $P L_{\kappa}(\mathbb{R})$ of all piecewise-linear homeomorphisms which have support in $[0,1]$ having break points contained in the set of dyadic rationals in $[0,1]$ and slopes contained in the multiplicative subgroup of $\mathbb{R}^{*}$ generated by 2 .

(iv) To prove (iv), recall that Grabowski [8] has shown that the free group of rank $c$ the continuum embeds in the group of compactly supported $C^{k}$ diffeomorphisms $(1 \leq k \leq \infty)$ of any positive-dimensional manifold. In particular, this is true of 
$\operatorname{Diff}\left(\mathbb{S}^{1}\right)$. It follows easily that $\widetilde{\operatorname{Diff}}\left(\mathbb{S}^{1}\right)$ also contains a free group of rank the continuum. By part (i), this completes the proof.

Lemma 3.1. The group $Q I^{+}(\mathbb{R})$ is torsion-free.

Proof. Let $f \in P L_{\delta}(\mathbb{R})$ be such that $[f] \neq 1 \in Q I^{+}(\mathbb{R})$. Thus $f-i d$ is unbounded. Choose a sequence $\left(a_{n}\right)$ of real numbers such that $a_{n} \rightarrow+\infty$ as $n \rightarrow+\infty$ and $\left|f\left(a_{n}\right)-a_{n}\right| \rightarrow+\infty$. Let $k>1$ be any integer. Suppose that $f\left(a_{n}\right)>a_{n}$. Since $f$ is order preserving, for each $n$ we have $a_{n}<f\left(a_{n}\right)<\cdots<f^{k}\left(a_{n}\right)$. In particular $f^{k}\left(a_{n}\right)-a_{n}>f\left(a_{n}\right)-a_{n}$. Similarly, $a_{n}-f^{k}\left(a_{n}\right)>a_{n}-f\left(a_{n}\right)$ in case $a_{n}>f\left(a_{n}\right)$. Therefore $\left|f^{k}\left(a_{n}\right)-a_{n}\right|>\left|f\left(a_{n}\right)-a_{n}\right| \forall n$ and hence $f^{k}-i d$ is unbounded. Hence $\left[f^{k}\right] \neq 1$ in $Q I^{+}(\mathbb{R})$ for $k>1$.

Remark 3.2. Thompson's group $G$ does not imbed in $Q I(\mathbb{R})$ since it has an element of order 3, whereas it follows from Lemma 3.1 that all torsion elements in $Q I(\mathbb{R})$ are of order 2 .

\section{ACKNOWLEDGEMENTS}

Part of this work was done while the author was visiting the University of Calgary, Alberta, Canada, during the spring and summer of 2003. It is a pleasure to thank Professors K. Varadarajan and P. Zvengrowski for their invitation and hospitality as well as financial support through their NSERC grants, making this visit possible.

\section{REFERENCES}

1. M.R. Bridson and A. Haefliger, Metric spaces of non-positive curvature, Grund. Math. Wiss., 319, (1999), Springer-Verlag, Berlin. MR1744486 (2000k:53038)

2. M. Brin and C.C. Squier, Groups of piecewise linear homeomorphisms of the real line, Invent. Math., 79, (1985), 485-498. MR0782231 (86h:57033)

3. K.S. Brown and R. Geoghegan, An infinite dimensional torsion-free $F P_{\infty}$ group, Invent. Math. 77, (1984), 367-381. MR0752825 (85m:20073)

4. J.W. Cannon, W.J. Floyd, and W.R. Parry, Introductory notes on Richard Thompson's groups, Enseign. Math. 42, 215-256, (1996). MR1426438 (98g:20058)

5. J. Dydak, A simple proof that pointed connected FANR-spaces are regular fundamental retracts of ANR's. Bull. Polon. Acad. Sci. Ser. Sci. Math. Astron. Phys., 25, (1977), 55-62. MR.0442918 (56:1293)

6. P. Freyd and A. Heller, Splitting homotopy idempotents-II, J. Pure Appl. Algebra, 89, (1993), 93-106. MR1239554 (95h:55015)

7. M. Gromov, Infinite groups as geometric objects, Proc. ICM, Warsaw, 1982-1983. MR0804694 (87c:57033)

8. J. Grabowski, Free subgroups of diffeomrphism groups, Fund. Math. 131, (1988), 103-121. MR 0974661 (90b:58031)

9. M. Imbert, Sur l'isomorphisme du groupe de Richard Thompson avec le groupe de Ptolémée Geometric Galois actions, 2, 313-324, London Math. Soc. Lecture Note Ser., 243, Cambridge Univ. Press, Cambridge, 1997. MR.1653017(99k:20078)

10. R. McKenzie and R.J. Thompson, An elementary construction of unsovable word problems in group theory, Word problems W.W. Boone et al., (eds.), Studies in Logic and the Foundations of Mathematics, 71, 457-478, North-Holland, Amsterdam, 1973. MR0396769 (53:629)

Institute of Mathematical Sciences, Cit Campus, Taramani, Chennai 600 113, India

E-mail address: sankaran@imsc.res.in 